\title{
Optimization and incorporating of green traffic for dynamic vehicle routing problem with perishable products
}

\author{
Reza Zakaryaei Talouki ${ }^{1} \cdot$ Nikbakhsh Javadian $^{2} \cdot$ Mohammad Mehdi Movahedi $^{1}$
}

Received: 31 July 2020 / Accepted: 16 February 2021 / Published online: 10 March 2021

(C) The Author(s), under exclusive licence to Springer-Verlag GmbH, DE part of Springer Nature 2021

\begin{abstract}
In view of the significance of transportation management and logistics in the economic concept and raising the productivity of production systems, well-timed procurement of perishable materials and goods is determined as a pivotal prerequisite for economic and environmental development. Since the perishable goods produced must be made delivered to consumers as early as possible on account of the limited lifespan, thus, the vulnerability of these products is extremely high, owing to the high cost of transportation as well as the environmental impacts. So that solves this problem, this study represents a problem of dynamic green vehicle routing of perishable products in green traffic conditions that optimizes the total cost for a dynamic transportation network and minimizes environmental influences, and increases customer satisfaction. The introduced model is implemented in light of time windows as a trustworthy solution for monitoring the dynamic logistics process and attaining instantaneous information on the basis of the green traffic situation and travel duration, which is commonly known by the Logit function. Assuming the threeobjective programming model, we consider a new improved algorithm developed for a novel augmented $\varepsilon$-constraint heuristic approach. Furthermore, robust optimization has been conducted for the established problem to tackle with uncertainties. Uncertainties are included demand and economic parameters. Eventually, to validate the proposed model, a case study was carried out at Kaleh Amol Dairy Company in Iran. The conclusions of sensitivity analysis by implementing the model in the real world indicate that the model and approach presented in various uncertainty scenarios have high flexibility.
\end{abstract}

Keywords Robust optimization · Dynamic green vehicle routing problem (DGVRP) · Augmented $\varepsilon$-constraint $\cdot$ Perishable products $\cdot$ Green traffic

\section{Introduction}

In recent decades, one of the outstanding issues that accomplish a substantial role in the success of companies to permanence in a competitive market is the design of a distribution network in a supply chain. In this regard, it is essential to address managing the distribution of costs and minimizing them, managing routes, and macro-investments in marketing. Neglecting the use of these factors in transportation can lead to

Responsible Editor: Philippe Garrigues

Nikbakhsh Javadian

Nijavadian@ustmb.ac.ir

1 Department of Industrial Management, Firoozkooh Branch, Islamic Azad University, Firoozkooh, Iran

2 Department of Industrial Engineering, Mazandaran University of Science and Technology, Babol, Iran enhance in the price of goods or the cost of delivery if higher incomes are achieved. In this case, the sales volume may decrease or the customer may take over from another product, which causes a decrease in customer trust.

Furthermore, with the aiming of raising efficiency in transportation and logistics systems, the problem of vehicle routing and suggesting reliable and efficacious solutions to give services to customers has been proposed and implemented. The famous VRPs (Huang et al. 2019; Rezgui et al. 2019) that first of all improve the economic impact of vehicle routes in the distribution of goods. Hence, in this work, have considered two types of VRP, which include green VRP and VRP for perishable products (Xu et al. 2019; Utama et al. 2020).

The crucial challenges of the century are global warming and greenhouse gas emissions (GHG). Accordingly, scholars have constantly paid close attention to vehicle routing (VRP) to optimize environmental impact, since transportation is one of the most paramount components in pollutant production in greenhouse gas emissions (Sureeyatanapas et al. 2018). 
Today, for instance, about $21 \%$ of global carbon emissions are generated from transportation systems (Buhler and Jochem 2008; Jabali et al. 2012). Dynamic Green VRP (GVRP) models are established upon the increase in the cost of distribution of goods in a supply chain network and the extent of pollution engendered by vehicles. To this effect, in the classic VRP, we are seeking to optimize routes for the car that can effectually serve the customer and reduce the total cost of travel, however, in GVRP which is an incorporation of dynamic vehicle routing (DVRP) and the Green Vehicle routing problem(GVRP) (Herbon and Khmelnitsky 2017), so have attempted to minimize environmental pollution. Moreover, the significance of green supply chains (GVRP) has attracted the attention of plenty of authors like (Koc and Karaoglan 2016; Poonthalir and Nadarajan 2018; Tiwari and Chang 2015; Yin and Chuang 2016). Even though reducing the distance or cost has a significant influence on reaching low $\mathrm{CO} 2$ emissions, but, there are other crucial causes in transportation, containing vehicle speed, vehicle load, traffic congestion, and road slopes, which take the pivotal role to achieve the shortest route (Ma et al. 2017). In particular, activities like moving vehicles, stopping, and restarting them can besides affect carbon emissions (Dunn et al. 2012).

Every day, a large variety of perishable goods are made a delivered from suppliers to recipients. Meanwhile, some products, as an example of food and pharmaceuticals, have to move perishable products as rapidly as possible on account of their limited lifespan. In this vein, time constraints, high transport frequencies, and existing traffic conditions enhance the exorbitant costs in this system; hence, optimizing the transportation costs for the supply of perishable products considering the traffic is necessary for this industry. Thus, an outstanding amount of studies have accomplished on transportation systems for perishable products (Amorim and Almada-Lobo 2014; Govindan et al. 2014a, 2014b; Song and Ko 2016).

This paper fulfills a mathematical model for solving a vehicle routing problem, consisting of total carbon dioxide emissions from vehicles with and without consignment, and optimizing operating costs and product durability. Investigations have taken into consideration the effect of the transportation system on the environment, for instance, in the concept of road emissions and accidents (Ćirović et al. 2014; Erdoğan and Miller-Hooks 2012; Lin et al. 2014) have established research. Owing to the plenty of factors that have an impact on the emission of gases, involving the type of vehicle, activities, road slopes, and traffic, accordingly, the redundancy of transportation of perishable products and the supply of products for the progression of sustainability is undeniable. That being the case, the transport systems of perishable products perform a pivotal role in air pollution. In this context, dynamic transportation presents a dependable solution for controlling and organizing the transportation of perishable products.
Hence, this study intends to accomplish a DGVRP for perishable products with the purpose to minimize total cost and carbon emissions. Additionally, in this model, customer satisfaction is a substantial factor in connection with product durability and delivery duration.

In this study, to attain the probability and allocation of routes in accord with the traffic conditions throughout the travel time, a model of Logit function is conducted that makes the transportation dynamic. In particular, on the basis of each customer, there is a set period of duration at which the product must be received, so the delivery components must reach each customer in their specific time windows (Zulvia et al. 2020). In the majority of the research conducted in this field, the speed of vehicles is constantly presuming to be amid the demand nodes. As a matter, dynamic environmental factors like traffic fluctuate and after examining the gaps in this article, five various traffic modes (very heavy, heavy, balanced, light, and very light) with applying a new strong DGVRP along with time windows were considered for perishable products with travel time and unspecified demand (Rahbari et al. 2019), also securing the solution with a robust optimization approach and using a novel augmented $\varepsilon$-constraint method for our proposed single-objective model.

In the next section, the recent related literature is reviewed. In Section 3, the problem is formulated in detail. In Section 4, the solution approach is explained. Section 5 presents a real case study of a dairy company to illustrate the effectiveness of the proposed model. We make our conclusion in Section 6 and recommend several future directions along with this study.

\section{Literature review}

In the current section, a brief and comprehensive overview of the main points of some previous theories of VRP and DGVRP is examined, taking into consideration traffic, multi-objective optimization problems, and research toward perishable goods.

Today, there is an indisputable concern about the distribution of transportation costs in the majority of organizations and industries. As a matter of fact, on account of the complexity of product design and decision-making in real business environments, fundamental problems are increasingly seen in VRP research, containing government regulations, time constraints, and traffic conditions (Zulvia et al. 2020).

The problem of vehicle routing was primarily announced by Dantzig and Ramser (1959), which later became a crucial problem in distribution management. Famous VRPs (Huang et al. 2019; Rezgui et al. 2019) sought to make progress in the economic influence of vehicle routes on the distribution of goods. From the other point of view, transportation technologies play a noteworthy role in air pollution. In recent times, green procurement has emerged in the transportation issue, as 
discussed in some prior investigations (Marcilio et al. 2018; Sim 2017; Soysal et al. 2018; Xiao and Konak 2017).

GVRP is designed to balance environmental and economic costs. This is developed by discovering effective ways to address ecological and financial concerns in diverse models. At GVRP, shipping companies and their customers have a variety of concerns, consisting of decreasing carbon emissions in shipping companies, which has been the subject of much more research. In this regard, in the concise and extensive overview of the fundamental points of GVRP in the articles that fulfilled, it is worth mentioning that much more of the models are single-objective, including cost (Govindan et al. 2014a, 2014b., 2014; Macrina et al. 2019a, 2019b; Qian and Eglese 2016; Soleimani et al. 2018) and carbon emissions (Jabbarpour et al. 2015; Soleimani et al. 2018; Suzuki and Kabir 2015). In these cases, some of the possible paths between customers are considered constantly or the speed of vehicles is assumed independent of time (Xiao et al. 2019; Suzuki and Kabir 2015; Tiwari and Chang 2015; Vornhusen and Kopfer 2015) and the average vehicle speed in a variety number of articles (Xiao and Konak 2016; Zhang et al. 2015) is fulfilled differently over periods. On the other hand, a large number of investigations in GVRP as Xiao and Konak (2016), Jabbarpour et al. (2015), and Franceschetti et al. (2017) considered varying speeds. Moreover, plenty of models in GVRP (such as Kwon et al. 2013; Solano-Charris et al. 2015; Vornhusen and Kopfer 2015) are accomplished with a heterogeneous fleet of vehicles.

VRP about the concept of perishable products is a type of VRP that concentrates on the supply of perishable products. Experience has indicated that perishable products have a short shelf life and must be made a delivery before being destroyed. Some of the VRP contexts associated with perishable products can be found by (Amorim and Almada-Lobo 2014; Song and Ko 2016; Rahimi et al. 2017; Rezaei-Malek et al. 2016). Also, since one of the primary substantial factors for customers is the time of arrival of goods, companies must be accountable to their customers within a certain period, so the significance of a specific time window for receiving goods to the customer is undisputable (Pérez-Rodríguez and Hernández-Aguirre 2019; Pradenas et al. 2013; Qian and Eglese 2016). Hence, the delivery of goods outside of windows is subject to fines (Taş et al. 2014). The additional paramount common variant of the GVRP is that the restriction of the capacities of vehicles has been contemplated (Macrina et al. 2019a, 2019b; Vidal et al. 2013).

A myriad of type of time window based on GVRP is implemented by applying the heuristic methods (Bruglieri et al. 2019; Kucukoğlu et al. 2015; Niu et al. 2018; Pradenas et al. 2013; Qian and Eglese 2016; Xiao and Konak 2015; Giallanza and Puma 2020). Likewise, an abundance of heuristic algorithms is existent for the VRP systems (Solano-Charris et al. 2015) like the memory structure adjusted simulated metaheuristic algorithm adopted by Kucukoğlu et al. (2015).
The vehicle routing problems which fulfill a substantial role in proper coordination in the movement of goods are influenced by the time uncertainty because each vehicle is affected by the movement of another. A paper presented by (Tirkolaee et al. 2020, 2020, 2020) attempted to conduct the reliable Pollution-Routing Problem with Cross-dock Selection (PRP-CDS) where the outputs are processed and moved through at least one cross-dock. To develop the problem, a Bi-Objective MILP model is expanded, aiming to diminish the total cost containing pollution and routing costs and to raise supply reliability to achieve a sustainable supply chain. Owing to the high complicatedness of the problem, two outstanding meta-heuristic algorithms comprising the MultiObjective Simulated-annealing Algorithm (MOSA) and Non-dominated Sorting Genetic Algorithm II (NSGA-II) are formulated to give efficient Pareto solutions. The outcomes of real case study analysis demonstrate that the solution techniques can attain high-quality solutions and NSGA-II is contemplated as the most efficient solution tool and the optimal route planning. Alinaghian et al. 2020 was introduced as a MILP mathematical model for Green Inventory-Routing Problem with Time Windows (GIRP-TW) that employs a piecewise linearization technique to diminish the total cost comprising the cost of fuel consumption, driver cost, inventory cost, and usage cost of vehicles regarding diverse aspects. Next, to solve the problem, several meta-heuristic algorithms are formulated comprising the fundamental Tabu Search (TS) algorithms and Differential Evolution (DE) algorithm. The achieved outcomes illustrate that the augmented TS algorithm is an acceptable method to generate high-quality solutions. Babaee Tirkolaee et al. (2019) was established a MILP model to develop the sustainable multi-trip location-routing problem with time windows (MTLRP-TW) concerning medical waste management in the COVID-19 pandemic situation to decrease the traveling time, total violation from time windows/service importance, and total infection/environmental risk assessed on the population that living around disposal sites. In this situation, the time windows take advantage of a crucial role to clarify the preference of services for hospitals with a various range of risks. Moreover, a real-world case study was analyzed in Sari of Iran to assess the performance of the model, and eventually, numerous sensitivity analyses were accomplished to analyze the behavior of the objective functions. Anderluh et al. (2019) attempted to simulate different scenarios in the duration of the trip and carried out a solution in this context. The computational conclusions indicate that the rate of synchronization is straightly associated with the possible advancements with the reuse of the program. In the suggested multi-product, the multi-period model (Yavari and Geraeli 2019) was introduced by a closed-loop chain for perishable products in the dairy industry. Besides, the demand and rate of return are taken into consideration uncertain. Hence, a mixed linear integer planning (MILP) model is 
formulated to diminish environmental costs and pollutants. $\mathrm{Xu}$ et al. (2019) in their study looked at the issue of capacitive green vehicle routing (GVRP) at variable vehicle speeds and time windows. The outcomes suggest that fuel consumption in a supply chain can be drastically decreased without notable loss of customer satisfaction. Koyuncu and Yavuz (2019) constructed an integrated framework in the area of mixed fleet GVRP, which conducts three various refueling strategies regarding made shorter time. Conclusions exhibited that generally, the arc-duplicating formulation was established to diminish the total cost of traveling. An investigation by Babaee Tirkolaee et al. (2019) was conducted on a novel robust MILP model concerning a green vehicle routing problem with intermediate depots contemplating different urban traffic conditions, consumption of fuel, and time windows of services for perishable products. Continuously, to analyze and solve the established model, the CPLEX solver of GAMS software was utilized as an exact method. In the end, a case study problem is examined to assess the applicability of the model in real-world conditions employing sensitivity analyses. Niu et al. 2018 introduced the green open vehicle routing problem by taking into account time windows. In this case, the vehicles could not return to the depot. In comparison to the selection of closed routes, the open routes would carry out a reduction of total cost by $20 \%$. Xiao and Konak 2016 fulfilled a mixed-integer linear programming (MILP) model that permitted the vehicles to stop on arcs. Thus, the conclusions of the analysis announced that by applying this model, the emissions by about $8 \%$ are decreased. By presuming the traffic conditions given the decision variables, Qian and Eglese 2016 illustrated that the decreases in fuel emissions can be achieved by employing a distance-based criterion so that enhances the speed of vehicles in traveling. Owing to the significance of environmental issues, a recombination novel block approach is introduced by Tiwari and Chang 2015 regarding decreasing carbon emissions in the duration of the transportation of goods. SolanoCharris et al. 2015 in their investigation attempted to develop in the local search on the basis of meta-heuristics for the robust VRP with discrete scenarios to identify a set of routes so that reduces total cost. A low-carbon routing problem model by Zhang et al. 2015 introduced a combination of the carbon emission cost, vehicle usage cost, and fuel cost. Based on the route splitting solution, formulated an improved tabu search algorithm to deal with solving the problem. Govindan et al. 2014a, 2014b accomplished a two-echelon time windows model in the context of location-routing problem in the delivery system of perishable food established upon a hybrid meta-heuristics algorithm. A summary of the literature review can be found in Table 1.

Various articles have been published in the field of the vehicle routing problem in recent years but this study has been significantly different from recent studies.
To conclude, the main contributions of this study are summarized as follows:

- To the best of our knowledge, this is the first study that proposes a multi-objective robust optimization model to design a Dynamic Green Vehicle Routing Problem (DGVRP).

- Applying a hybrid method based on a heuristic algorithm and the augmented $\varepsilon$-constraint method to solve the problem.

- Consideration of product expiration time due to the contemplation of the lifetime of products for the time of delivery is of particular importance in the proposed model.

- Considering different traffic situations with time windows.

The Logit function is used to obtain probability and assign routes in the model.

\section{Methodology}

The study optimizes a DGVRP model for perishable products taken aimed at reducing total costs and carbon dioxide emissions, as well as raising customer service levels. In this presented model, there are limitations containing product durability, time windows for each customer, time dependence on account of a peak, and traffic hours. In this regard, we have taken account of a company that produces new products with low permanence that must be made a delivery to the destination before decay at the determinate time. It is additionally essential to mention that even though the product is stored in a refrigerated vehicle, but if it does not get to the destination on time, the quality of the product will decrease. This is because the door of the vehicle must be opened and closed when delivering the product to each customer and this factor has a fundamental influence on reducing the temperature, which takes one to a reduction in the durability and quality of the product (Song and Ko 2016). Furthermore, the paramount cause of air pollution is identified in the transportation system. Hence, the designed system makes an effort to reduce air pollution to reduce $\mathrm{CO} 2$ emissions. The production of greenhouse gases produced throughout a shortstop and the restart of the car while delivering or unloading the product to customers are other factors that have a significant impact on $\mathrm{CO} 2$ emissions (Gaines et al. 2013). In addition, to enhance customer satisfaction with the level of service, each customer is assigned a particular delivery time window, which will be to a greater extent satisfied if the customer takes in the product with the best quality within the indicated time. Thus, the proposed DGVRP model seeks to maximize customer satisfaction. 


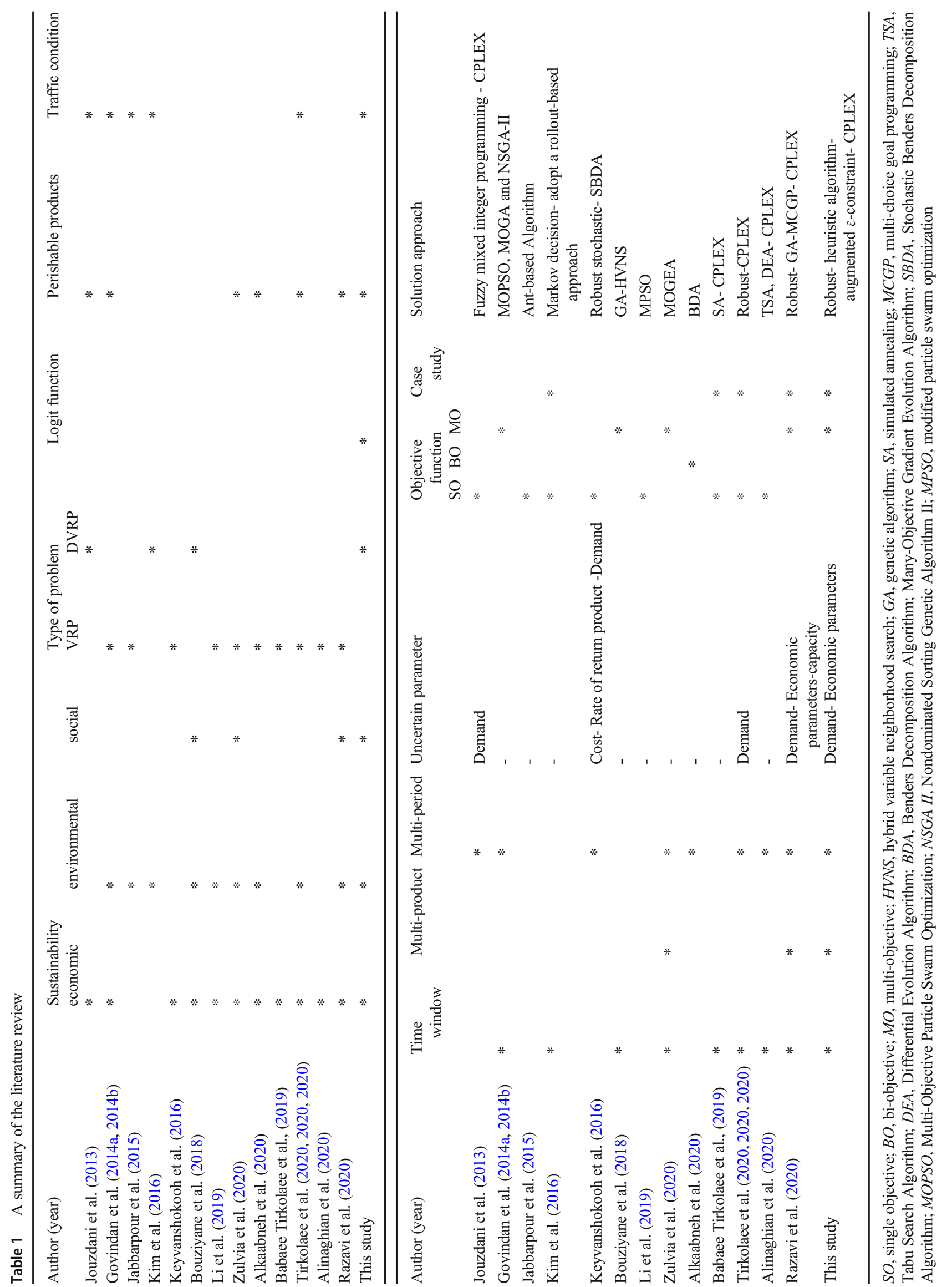


Since this study has three uncertain objective functions, robust optimization has been accomplished to solve this problem, and on the other hand, to solve the proposed multi-objective model, a heuristic combination method with an improved Epsilon method has been adopted.

\section{Proposed model}

In this section, in the beginning, the definitions, parameters, and decision variables are described; next, the proposed model is introduced with the definition of purposes and limitations.

Compared to the recent literature, this model has features such as considering the time window in traffic conditions, fuel consumption for the vehicle, and product quality according to product longevity, which addresses three objectives, economic, environmental, and social.

The hypotheses of the problem are as follows:

- Traffic congestion situation conforms a random process.

- The traffic situation determines the average speed of the vehicle.

- For each customer, a high limit of time to reach the destination is specified, and the customer wants to attain the desired destination up to that time. Therefore, if this does not happen, the customer's satisfaction with the service will decrease and the violation penalty will be considered excessive.

- The travel time is taken into account to be non-constant and dependent upon the speed and distance traveled.

- Demand, economic parameters are considered uncertain.

Sets and Indices: $N$ Sets of node, $i, j, c \in N$

CSets of customer, $c=i=\{1,2, \ldots, C\}$

$P$ Sets of product, $p=\{1,2, \ldots, P\}$

TSets of time period, $t=\{1,2, \ldots, T\}$

$V$ Sets of vehicle, $v=\{1,2, \ldots, V\}$

Parameters $d i s_{i j}$ The distance between node $i$ and $j$

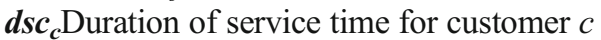

$\boldsymbol{m} s_{i j t}$ Maximum speed allowed on the node $i$ to node $j$ route at time period $t$

$\boldsymbol{p s c}_{\boldsymbol{c} \text { 住 }}$ The primitive service time for customer $c$ to the node $n$ at time period $t$ $\boldsymbol{l} \boldsymbol{s c}_{\boldsymbol{c n t}}$ The latter service time for customer $c$ to the node $n$ at time period $t$

pst $t_{\text {cnt }}$ Primary service time for customerc to the node $n$ at time period $t$

$r s t_{c n t}$ Recent service time for customer $c$ to the node $n$ at time period $t$

et $v_{\boldsymbol{v}}$ The expecting time vehicle $v$ for customer $c$

$\boldsymbol{d} \boldsymbol{c} \boldsymbol{p}_{\boldsymbol{c p t}}$ The amount of demand of customer $c$ for product $p$ at time period $t$

$\boldsymbol{s c v} v_{v}$ The specified capacity of vehicle $v$

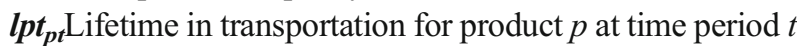

$\boldsymbol{p} v \boldsymbol{r}_{\boldsymbol{v}}$ Product quality diminution rate in vehicle $v$ this value is contingent upon the vehicle's type (refrigerated or nonrefrigerated).

$\operatorname{lr}_{p p t}$ Lifetime rate for product $p$ at time period $t$

$\boldsymbol{d} \boldsymbol{p} \boldsymbol{v}_{\boldsymbol{p} v \boldsymbol{t}}$ Deterioration rate of product $p$ make a delivery by vehicle $v$ at time period $t$

$a p t_{p t}$ Absence of quality cost for product $p$ at time period $t$

$\boldsymbol{c} v t_{v t}$ Cost of driver for vehicle $v$ at time period $t$

$f c v_{v t}$ Fuel cost for vehicle $v$ at time period $t$

$\boldsymbol{d} \boldsymbol{v} \boldsymbol{t}_{\boldsymbol{v c t}}$ The duration of door opening in vehicle $v$ ahead of serving customer $c$ at time period $t$

$\boldsymbol{d} \boldsymbol{r} \boldsymbol{v}_{\boldsymbol{v} j \mathrm{t}}$ The determined rate of Fuel consumption of vehicle $v$ while giving products from node $i$ to node $j$ at time period $t$ under traffic condition

$\boldsymbol{e v t _ { v }}$ Average amount of fuel used up per unit of distance by vehicle $v$ under traffic condition at time period $t$

$\boldsymbol{e} \boldsymbol{v}_{\boldsymbol{v} t}$ Empty-load vehicle $v$ at time period $t$

$f l v_{v t}$ Full-load vehicle $v$ at time period $t$

$\boldsymbol{g t t}_{\boldsymbol{v} j \mathrm{t}}$ Goods of vehicle $v$ as traveling from node $i$ to node $j$ at time period $t$

eil ${ }_{i j t}$ Volume of 2 emission so long as moving between node $i$ to node $j$ at time period $t$

$\boldsymbol{e} \boldsymbol{i} v_{\boldsymbol{v} t}$ Volume of 2 emission when the vehicle $v$ is restarted at time period $t$

$\boldsymbol{e i s}_{i j t}$ Volume of 2 emission at the beginning of start as traveling from node $i$ to node $j$ at time period $t$

$\beta$ Coefficient of conversion of gas emissions into costs

$M B i g$ number that equal to upper bound demand

Decision variable $\boldsymbol{s l} \boldsymbol{c}_{c t}$ Service level of customer $c$ at time period $t$

$t p c_{p c v t}$ Total corruption time of product $p$ make a delivery to customer $c$ by vehicle $v$ at time period $t$

$\boldsymbol{a t c}_{\boldsymbol{v} \text { cht }}$ Arrival time of vehicle $v$ for customer $c$ to the node $n$ at time period $t$

$\boldsymbol{d t} c_{v c n t}$ Departure time of vehicle $v$ for customer $c$ to the node $n$ at time period $t$ 
$\boldsymbol{X}_{\text {cvt }}$ If vehicle $v$ is assigned to customer $c$ for service at time period $t$, equal to 1 and otherwise 0

$\boldsymbol{Y}_{\text {cijvt }}$ If the $i$ to $j$ route is traveled by the vehicle $v$ to deliver the customer $c$, which starts at time period $t$, equal to 1 and otherwise 0

$Z_{i j v t}$ if vehicle $v$ is used for route $i$ to $j$ at time period $t$ equal to 1 and, otherwise 0

$\boldsymbol{U}_{\boldsymbol{v} c t}$ If the vehicle $v$ on during waiting and serving the customer $c$ at time period $t$ equal to 1 and, otherwise 0

$$
\begin{aligned}
& \min Z_{1}=\sum_{i \in \mathbf{E}} \sum_{j \in \mathbf{J}} \sum_{c \in \mathbb{C}} \sum_{v \in \mathbf{V}} \sum_{t \in \mathbf{T}}\left[\left(\frac{d i s_{i j}}{d r v_{v j i t} \cdot m s_{i j t}}\right)+\left(\left(d s c_{c}+e t v_{v c}\right) \cdot e v t_{v t}\right) \cdot X_{c v t}\right] . f c v_{v r} \cdot Y_{c i j v t}+ \\
& \sum_{i \in \mathbf{I}} \sum_{j \in \mathbf{J}} \sum_{v \in \mathbf{V}} \sum_{t \in \mathbf{T}} c v t_{v} \cdot Z_{i j v}+\sum_{p \in \mathbf{P}} \sum_{c \in \mathbf{C}} \sum_{v \in \mathbf{V}} \sum_{t \in \mathbf{T}} t p c_{p c t} \cdot d c p_{c p t} \cdot a p t_{p t}+\beta . \\
& {\left[\sum_{i \in \mathbf{I}} \sum_{j \in \mathbf{J}} \sum_{v \in \mathbf{V}} \sum_{t \in \mathbf{T}} d i s_{i j} \cdot\left(e i i_{i j t}+e i i_{j i t}\right)+e i v_{v t}\right]}
\end{aligned}
$$

$$
\begin{aligned}
\min Z_{2}= & \sum_{i \in \mathbf{I}} \sum_{j \in \mathbf{J}} \sum_{c \in \mathbf{C}} \sum_{v \in \mathbf{V}} \sum_{t \in \mathbf{T}}\left[e i l_{i j t} \cdot d i s_{i j} \cdot m s_{i j t} \cdot\left(e l v_{v t}+\left(\frac{f l v_{v t}-e l v_{v t}}{s c v_{v}}\right) \cdot g t t_{v i j t}\right)+\left(\left(d s c_{c}+e t v_{v c}\right) \cdot e v t_{v t}\right) \cdot X_{c v t}+\left(1-X_{c v t}\right) \cdot e i v_{v t}\right] \cdot Y_{c i j v t} \\
& +\sum_{i \in \mathbf{I}} \sum_{j \in \mathbf{J}} \sum_{v \in \mathbf{V}} \sum_{t \in \mathbf{T}} e i s_{i j t} \cdot Z_{i j v t}
\end{aligned}
$$

$\max Z_{3}=\frac{1}{N} \cdot \sum_{c \in \mathbf{C}} \sum_{t \in \mathbf{T}} s l_{c t}$

Subject to:

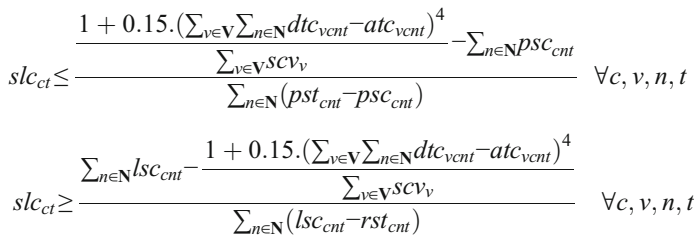

$\sum_{c \in \mathbf{C}} \sum_{i \in \mathbf{I}} \sum_{v \in \mathbf{V}} \sum_{t \in \mathbf{T}} Y_{c i j v t}=1_{\forall j \in N}$

$\sum_{c \in \mathbf{C}} \sum_{j \in \mathbf{J}} \sum_{v \in \mathbf{V}} \sum_{t \in \mathbf{T}} Y_{c i j v t}=1_{\forall i \in N}$

$Y_{c i j v t} \leq Z_{i j v t} \quad \forall i, j, v, t$

$\sum_{i \in \mathbf{I}} Y_{c i j^{\prime} v t}-\sum_{j \in \mathbf{J}} Y_{c j i v t}=0_{\forall c, v, t, j^{\prime} \in N}$

$\sum_{j \geq 2 \in \mathbf{J}} \sum_{c \in \mathbf{C}} Y_{c 0 j v t}=\sum_{j \in \mathbf{J}} Z_{i j v t} \forall v, t, i \in N$

$\sum_{i \geq 2 \in \mathbf{I}} \sum_{c \in \mathbf{C}} Y_{c i 0 v t}=\sum_{i \in \mathbf{I}} Z_{i j v t} \forall v, t, j \in N$

$\sum_{i \in \mathbf{I}} \sum_{j \in \mathbf{J}} Y_{c i j v t} \cdot d c p_{c p t} \leq \sum_{i \in \mathbf{I}} \sum_{j \in \mathbf{J}} Z_{i j v t} . s c v_{v} \forall c, p, v, t$

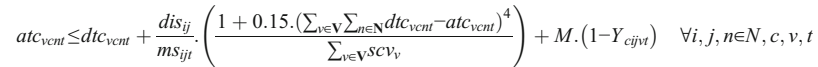

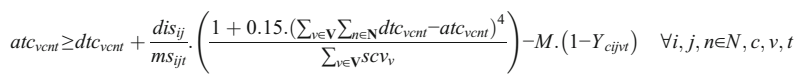

$d t c_{v c n t}=\left(a t c_{v c n t}+d s c_{c}\right) \cdot U_{v c t} \quad \forall c, v, n, t$

atc $_{v c n t} \geq p s c_{c n t} \quad \forall c, v, n, t$

$d t c_{v c n t} \leq l s c_{c n t} \quad \forall c, v, n, t$

$t p c_{p c v t}=\left(p v r_{v} \cdot l r p_{p t} \cdot a t c_{v c n t}\right)+\left(d p v_{p v t} \cdot d v t_{v c t} \cdot d s c_{c}\right) \quad \forall c, v, n, p, t$

$t p c_{p c v t} \leq l p t_{p t} \quad \forall p, c, v, t$

$U_{v c t}, Y_{c i j v t}, Z_{i j v t}, X_{c v t} \in\{0,1\} \quad \forall c, i, j, v, t$

$\operatorname{slc}_{c t}, t p c_{p c v t}, a t c_{v c n t}, d t c_{v c n t} \geq 0 \quad \forall p, c, v, n, t$

Equation (1) shows the function of the first objective of the problem, which is to minimize costs, including fuel costs, vehicle costs, cost estimates for product quality degradation, and environmental costs. Equation (2) shows the function of the second objective of the problem, which is to minimize the environmental impacts, which includes examining the amount of carbon emissions in the path is moving between the two 
nodes, at the start of the vehicle and while waiting to be unloaded. Equation (3), which shows the function of the third objective of the problem, shows the maximization of the level of customer service based on a function of time that is considered according to the time window of the customer service level. Constraints (4) and (5) show the level of customer service with concerning the logit function in the time window. These equations express the level of service at the time of arrival and time of departure according to traffic conditions, which shows the best level of service to customers at the time of initial service and recent time of service due to the random distribution of service time. Constraints (6) and (7) ensure that each route is visited by the customer only once. Constraint (8) indicates that customers are only served if the vehicle is active. Constraint (9) is the restriction of route formation. Constraints (10) and (11) show the relationship between incoming and outgoing vehicles to serve customers in the same time period for selected routes. Constraint (12) shows the relationship between the amount of demand and the capacity of vehicles. Constraints (13) to (14) estimate the time it takes for each node to reach the vehicle to transport the customer on the route, at which time the distance traveled on each route depends on the speed of the vehicle. Constraint (15) calculates the departure time. Constraints (16) and (17) are the upper and lower limits of the time window that determine the level of customer service. Constraint (18) is the calculation of total corruption time of product makes a delivery to customer by vehicle during the time period. Constraint (19) shows the relationship between the total corruption time of product and the lifetime of products. Constraints (20) to (21) display the types of variables.

It should be noted that the proposed model is an NP-hard one because it is the extended form of the vehicle routing problem that is already NP-hard. It means that the required solution time increases exorbitantly with size. Also, the general size of the problem is as follows: the proposed model contains $(C \times T)+(P \times C \times V \times T)+(V \times C \times N \times T)+(V \times$ $C \times N \times T)+(C \times V \times T)+(C \times I \times J \times V \times T)+(I \times J \times V \times$ $T)+(V \times C \times T)$ decision variables and also contains $(C \times V \times$ $N \times T)+(C \times V \times N \times T)+(J)+(I)+(I \times J \times V \times T)+(C \times$ $V \times J \times T)+(V \times J \times T)+(V \times T \times I)+(V \times T \times J)+(C \times P \times$ $V \times T)+(I \times J \times N \times C \times V \times T)+(I \times J \times N \times C \times V \times T)+$ $3 \times(C \times V \times N \times T)+(C \times V \times N \times P \times T)+(P \times C \times V \times T)$ constraints.

\section{Robust optimization}

This research applies the scenario-based robust optimization method to cope with uncertainty. The main reason for using this approach is as follows. Based on Bairamzadeh et al. (2018), there are three types of uncertainty namely (a) randomness, (b) epistemic, and (c) deep uncertainty. Randomness uncertainty occurs when there is enough historical data to estimate the probability distribution function of the parameter. Epistemic uncertainty is related to a lack of knowledge about the values of the parameters. In this regard, expert opinion is gathered in the linguistic way to estimate the probability distribution function of the parameter. Eventually, deep uncertainty is related to a lack of information about input data. As discussed in Bairamzadeh et al. (2018), to tackle randomness uncertainty, a (robust) scenario-based optimization method can be employed. Hence, due to the condition of the current study, the research problem is investigated under randomness uncertainty and scenario-based robust optimization is applied to tackle uncertainty. On the other side, the scenario-based stochastic programming model allows the model to consider different quantities for uncertain parameters through a finite number of scenarios; hence, the model determines the optimal objective value according to each scenario's occurrence likelihood.

As result, due to the uncertainties in demand and economic parameters in the proposed model, robust optimization is used to address this problem. The robust optimization model of the proposed problem is shown below (Gholizadeh et al. 2020, 2020; Gholizadeh and Fazlollahtabar 2020).

$$
\begin{aligned}
\operatorname{Min} Z F= & \sum_{s} P_{s} Z_{1_{s}}+\lambda \sum_{s} P_{s}\left(Z_{1_{s}}-\sum_{s^{\prime}} P_{s^{\prime}} Z_{1_{s^{\prime}}}+2 \theta 1_{s}\right) \\
& +\omega \sum_{s} P_{s} \delta_{s}
\end{aligned}
$$

$\operatorname{Min} Z E=\sum_{s} P_{s} Z_{2 s}+\lambda \sum_{s} P_{s}\left(Z_{2 s}-\sum_{s^{\prime}} P_{s^{\prime}} Z_{2 s^{\prime}}+2 \theta 2_{s}\right)$

$\operatorname{Max} Z G=\sum_{s} P_{s} Z_{3 s}-\lambda \sum_{s} P_{s}\left(Z_{3 s}-\sum_{s^{\prime}} P_{s^{\prime}} Z_{3_{s^{\prime}}}+2 \theta 3_{s}\right)$

S.t

$Z_{1 s}-\sum_{s^{\prime}} P_{s^{\prime}} Z_{1_{s^{\prime}}}+\theta 1_{s} \geq 0 \quad \forall s$

$Z_{2 s}-\sum_{s^{\prime}} P_{s^{\prime}} Z_{2 s^{\prime}}+\theta 2_{s} \geq 0 \quad \forall s$

$Z_{3 s}-\sum_{s^{\prime}} P_{s^{\prime}} Z_{3 s^{\prime}}+\theta 3_{s} \geq 0 \quad \forall s$

$\theta 1_{s}, \theta 2_{s}, \theta 3_{s} \geq 0 \quad \forall s$

and constraint (4)-(29)

Where $\lambda$ is the weight of risk and $\omega$ is the infeasibility weight being set in an experimentally manner by the decision-maker and $\mathrm{P}_{s}$ is the probability of scenario $\mathrm{s}$ and $\mathrm{P}_{s^{\prime}}$ is the probability of scenario $s^{\prime}$ that $s^{\prime} \in s . \theta_{s}$ is the linearization variable under the scenario $s$. Also, $Z_{1 s}, Z_{2 s}$ and $Z_{3 s}$ is objective functions under the scenario $s$.

The first and second terms in Eqs. (22), (23), and (24) indicate mean value and variance of the objective functions, 
respectively, and the last terming of the objective function (22) is to measure the model robustness in terms of the infeasibility values of control constraints under each scenario. The constraint (25), (26), and (27) is auxiliary constraints added to the model for converting the nonlinear objective function linearly, and the constraint (28) is non-negative variables.

\section{Solution approach}

As previously mentioned, the model presented in this paper uses three objective functions with different directions (a minimization function and a maximization function). Therefore, this requires establishing interactivity between the target functions. According to the models have three conflicting objectives and finding a globally optimal solution is not possible. In that case, the Pareto-optimal/non-dominated/non-inferior/efficient solution concept is required. In the case of finding a Pareto-optimal (feasible) solution to multi-objective models, the generation of efficient responses and subsequent decision-making conflicts when all the answers are prepared as far as, in this research, we employ an augmented $\varepsilon$-constraint method.

\section{Augmented $\varepsilon$-constraint method}

Due to the structure of this research which has considered a real case study, applying multi-objective solving methods, which give a Pareto front, the method modifies the constraint of the pivotal justified area and seeks angular solutions, may lead to some efficacious for firm's managers or decisionmakers as the managers of the emergency operation centers usually expect to face a determined solution to plan for inventory condition. Hence, we have applied a Pareto-based algorithm, then, we have to use a method to select several of the Pareto solutions to present to managers and decision-makers. As a matter of fact, the steps of the $\varepsilon$-constraint method are matching by Gholizadeh et al. 2020, 2020.

The following algorithm is employed in this method using Eqs. (29) to (31)

$$
\operatorname{Max}\left(f_{1}(x)+e p s \times\left(\frac{s_{2}}{r_{2}}+10^{-1} \frac{s_{3}}{r_{3}}+\ldots+10^{-(p-2)} \frac{s_{p}}{r_{p}}\right)\right)
$$

Subjected to:

$$
X \in F
$$

$k=2, \ldots, p f_{k}(X)-S_{k}=e_{k}(31)$

$k=2, \ldots, p e_{k}=l b_{k}+i_{k} * \operatorname{step}_{k}(31)$

$f_{k}(X)$ Main objective functions of the model to be optimized $l b_{k}$ The lower bound for the objective function $k$

step $_{k}={ }^{r} k / g_{k}$ Step size of the objective function $k$

$r_{k}$ Variation range of objective function $k$

$g_{k}$ The number of intervals needed for the objective function $k$

$S_{k}$ The surplus variable for the objective function $k$

$F$ Acceptable range

eps A very small number (usually $10^{-3}-10^{-6}$ )

\section{Heuristic solution}

In this section, we consider a heuristic solution to the proposed model with the augmented $\varepsilon$-constraint method to relax the binary variable is used to solve the developed model efficiently. Since the computational time for each model of mixedinteger nonlinear programming (MINLP) and mixed-integer linear programming (MILP) increases with increasing variables and the presence of data, as a result, over time, even in some cases, there is no acceptable solution. Therefore, an exploratory method based on the relaxation of a binary variable is proposed. First, we consider the binary variable greater than zero and solve the optimization model from the optimal solutions we consider all non-zero binary variables and as a new constraint, we add a mixed-integer to the nonlinear model and we solve again the optimization model. The main advantages of this method are that it leads to a drastic reduction in the CPU time of solving the problem and also can obtain highquality solutions. On the other hand, the disadvantage of this method is that it sometimes sticks in the loop and cannot obtain the solution.

The steps of this method are as follows:

Step 1: Release Constraints Zero and One by converting the proposed binary variables to a continuous positive variable. Solve the released model

Step 2: Report or record all non-zero values for the released variable

Step 3: Set any non-zero value of the released variables to 1 and set them as constraints in the original MILP model. Solve the model again.

The pseudo code for suggested heuristic method is shown in Fig. 1 (Gholizadeh et al. 2020, 2020).

\section{Numerical example}

In this section, to evaluate the developed model, we consider various numerical experiments based on a real case study of a dairy company in Iran. The primary sources of data used in this study were from the Kaleh Company in Iran. Due to company-specific policies (non-disclosure of data) after collecting data from different sections of the dairy company and statistical analysis of the collected data, we obtained the distribution of the data behavior by statistical software, we use 
Fig. 1 Pseudo code for suggested heuristic method

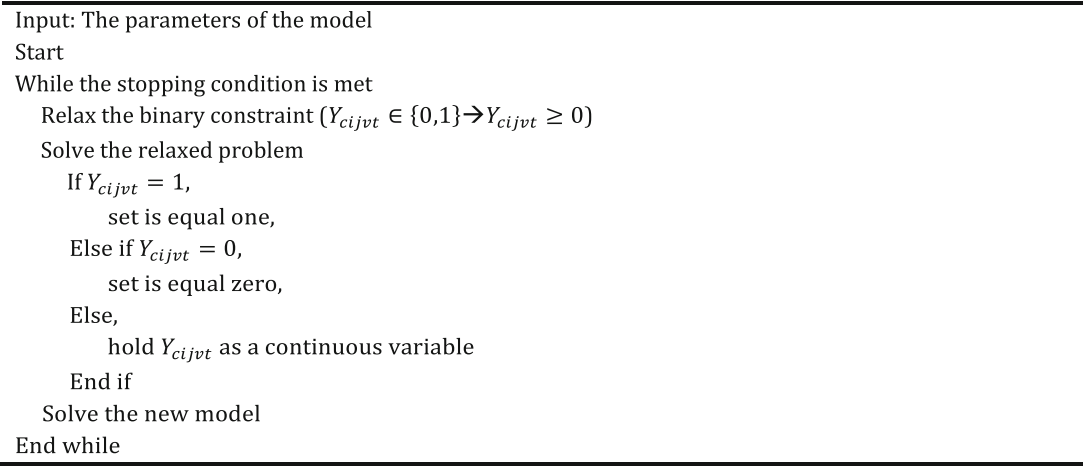

random distribution to implement the proposed model but this distribution is based on real data of Kaleh Company. Table 2 shows generate real data according to behavior Kaleh Company data. Based on the experiments accomplished to validate the proposed model, the results indicated the validity of the model, which has led to the extension of a solution that can be extended to other industries to support managers' decision-making with the heuristic.

The suggested mathematical model was coded in the GAMS 2017 software with solver CPLEX. Each test problem was solved using the developed heuristic and robust model according to the parameters based on uncertain experiments for three trials and the random distributions.

\section{Validating suggested model}

In order to evaluate the performance of the proposed model, 3 test problems with different sizes are developed and shown in Table 3. Tables 4, 5, and 6 illustrate the values of the proposed single objective function for each of the 3 different problem sizes. As can be seen in Tables 4, 5, and 6, the solution time for the three heuristic methods and MILP and MINLP is different in deterministic and robust states, which by comparing them shows that the heuristic method reduces solution time by approximately $27 \%$ and $15 \%$ compared to the MINLP and MILP and it shows efficiency and proper performance of the proposed method.

As can be seen in Tables 4, 5, and 6 and Fig. 2, the value of the objective functions increases when the size of the problem increases. Moreover, the objective function value in the deterministic condition is lower than the robust condition, which demonstrates the proper performance of the model in dealing with uncertainty. Considering the outputs for the three solution methods, the performance of the heuristic approach is higher than two other approaches based on the objective function value and the time to solve the problem.

\section{Study results with the proposed solution}

According to Section 4, as stated above, the augmented $\varepsilon$ constraint method is integrated with the heuristic method, according to Section 5, which is a small part of case study including 1ID, 13 customers, and 3 vehicles and the output of the proposed model with the obtained Pareto solutions for the deterministic and robust state is shown below. One of the main applications of the present study proves that how much the results can be different in the real world is the comparison of obtained results of deterministic and robust problems.

According to Fig. 3, the results of the model and the proposed method have reduced the total cost and environmental

Table 2 Generate real data according to behavior Kaleh Company data

\begin{tabular}{|c|c|c|c|c|c|}
\hline Parameter & $\begin{array}{l}\text { Corresponding } \\
\text { random distribution }\end{array}$ & Parameter & $\begin{array}{l}\text { Corresponding } \\
\text { random distribution }\end{array}$ & Parameter & $\begin{array}{l}\text { Corresponding } \\
\text { random distribution }\end{array}$ \\
\hline$d i s_{i j}$ & Uniform $(20,650)$ & $l r p_{p t}$ & Uniform $(0.5,0.7)$ & $g_{t} t_{\text {vijt }}$ & Uniform $(20,60)$ \\
\hline$d s c_{c}$ & Uniform $(20,100)$ & $d p v_{p v t}$ & Uniform $(0.5,0.7)$ & $e i l_{i j t}$ & Uniform $(5,15)$ \\
\hline$p s c_{c n t}$ & Uniform $(50,150)$ & $a p t_{p t}$ & Uniform $(15000,50000)$ & $e i v_{v t}$ & Uniform $(2,10)$ \\
\hline$l s c_{c n t}$ & Uniform $(30,120)$ & $c v t_{v t}$ & Uniform $(10000,30000)$ & $e i s_{i j t}$ & Uniform $(5,15)$ \\
\hline$r s t_{c n t}$ & Uniform $(20,100)$ & $f c v_{v t}$ & Uniform $(5000,150000)$ & $\beta$ & Uniform $(0.3,0.5)$ \\
\hline$e t v_{v c}$ & Uniform $(150,350)$ & $d v t_{v c t}$ & Uniform $(15,30)$ & $M$ & 1000000 \\
\hline$d c p_{c p t}$ & Uniform $(250,850)$ & $d r v_{v i j t}$ & Uniform $(0.3,0.5)$ & $f l v_{v t}$ & Uniform $(50,350)$ \\
\hline$s c v_{v}$ & Uniform $(150,450)$ & $e v t_{v t}$ & Uniform $(15,150)$ & $p v r_{v}$ & Uniform $(0.1,0.5)$ \\
\hline$l p t_{p t}$ & Uniform $(5,10)$ & $e l v_{v t}$ & Uniform $(10,150)$ & $m s_{i j t}$ & Uniform $(20,160)$ \\
\hline
\end{tabular}


impacts, on the other hand, have increased customer satisfaction. The optimal solution generally resulted in a $14.45 \%$ reduction in total costs and $8.15 \%$ in environmental impacts, as well as a $2.18 \%$ increase in customer satisfaction. In addition, the uncertainty effect will directly lead to an increase in the number of vehicles in the company's routing process.

Figure 4 shows the impact of traffic conditions on the issue. As you can see, the traffic situation has led to an increase in total costs and environmental impacts and a decrease in customer satisfaction. Also, in non-traffic conditions, it has led to a $6.6 \%$ reduction in total costs in the deterministic state and a $40.9 \%$ decrease in the total cost in the robust state, which can be considered as large savings. On the other hand, it has led to a $0.73 \%$ reduction in environmental impacts in the deterministic state and a $24.9 \%$ reduction in the environmental impact in the robust state. Also, in non-traffic conditions, it has led to an $11 \%$ reduction in customer satisfaction in the deterministic state and a $42.5 \%$ decrease in the customer satisfaction in the robust state.

In other words, with the calm of traffic conditions, vehicles will have more opportunities to provide services to customers. This fact indicates the importance of planning traffic conditions in management decisions.

According to Fig. 5, in different modes of the optimal solution, it can be easily seen that the proposed model improves the company's costs by $12.6 \%$ in the deterministic mode, also improves the environmental effects by $7.5 \%$ and customer satisfaction by $2.18 \%$. However, we are in robust mode due to the existence of a penalty cost with a relatively small increase in costs.

\section{Sensitivity analyses}

This section is dedicated to analyze the impact of some parameters on the model. To achieve this goal, we solve the problem under different modes and analyze the obtained results.

\section{Demand}

In this section, we look at the effect of demand changes on the objectives of the proposed problem. As you can see in Fig. 6, with a $40 \%$ increase in demand, the total cost increases by

Table 3 Problem size

\begin{tabular}{ll}
\hline Problem number & Size of the problem \\
\hline 1 & $|\mathrm{i}| \times|\mathrm{j}| \times|\mathrm{c}| \times|\mathrm{p}| \times|\mathrm{v}| \times|\mathrm{t}|=3 \times 5 \times 3 \times 3 \times 2 \times 3$ \\
2 & $|\mathrm{i}| \times|\mathrm{j}| \times|\mathrm{c}| \times|\mathrm{p}| \times|\mathrm{v}| \times|\mathrm{t}|=5 \times 8 \times 5 \times 3 \times 3 \times 5$ \\
3 & $|\mathrm{i}| \times|\mathrm{j}| \times|\mathrm{c}| \times|\mathrm{p}| \times|\mathrm{v}| \times|\mathrm{t}|=8 \times 12 \times 8 \times 6 \times 6 \times 8$ \\
4 & $|\mathrm{i}| \times|\mathrm{j}| \times|\mathrm{c}| \times|\mathrm{p}| \times|\mathrm{v}| \times|\mathrm{t}|=10 \times 12 \times 10 \times 8 \times 8 \times 10$ \\
\hline
\end{tabular}

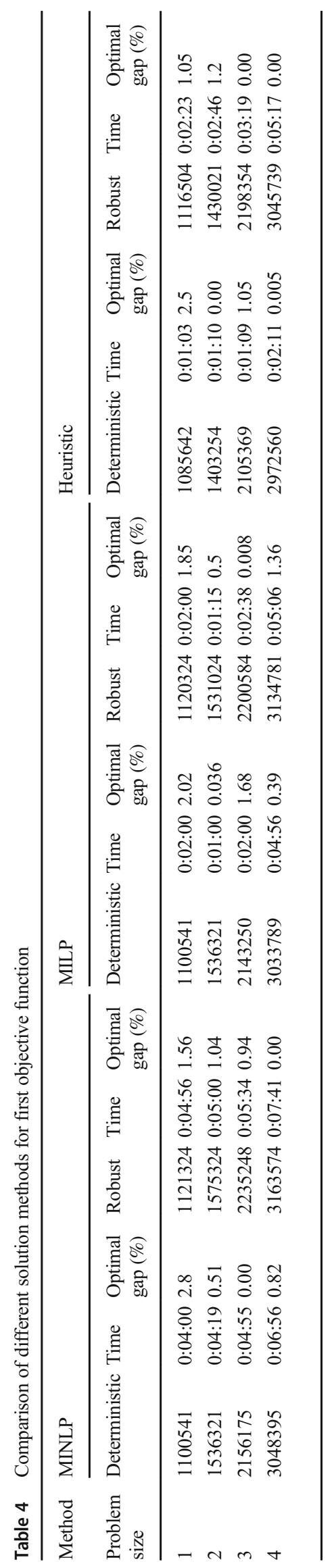



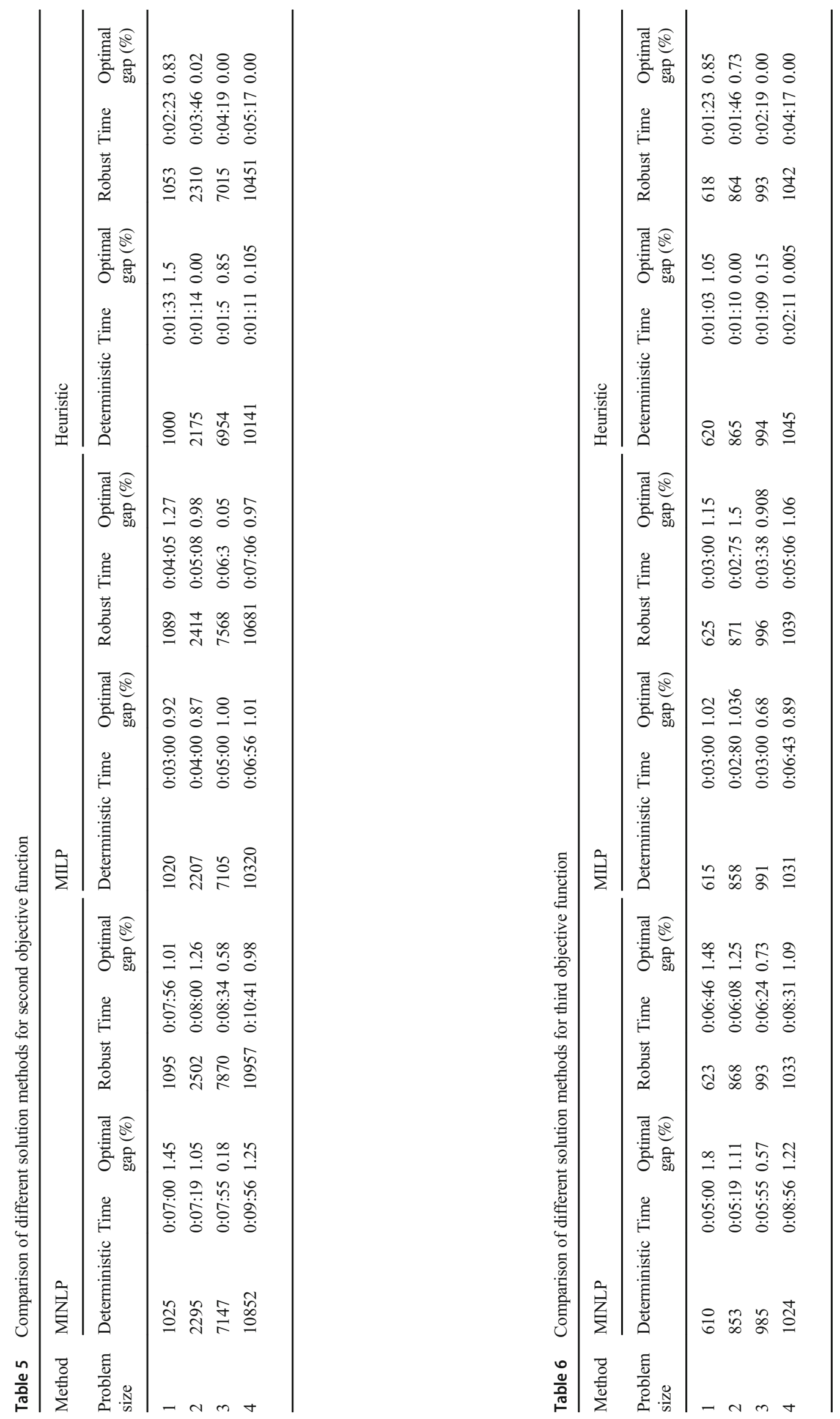


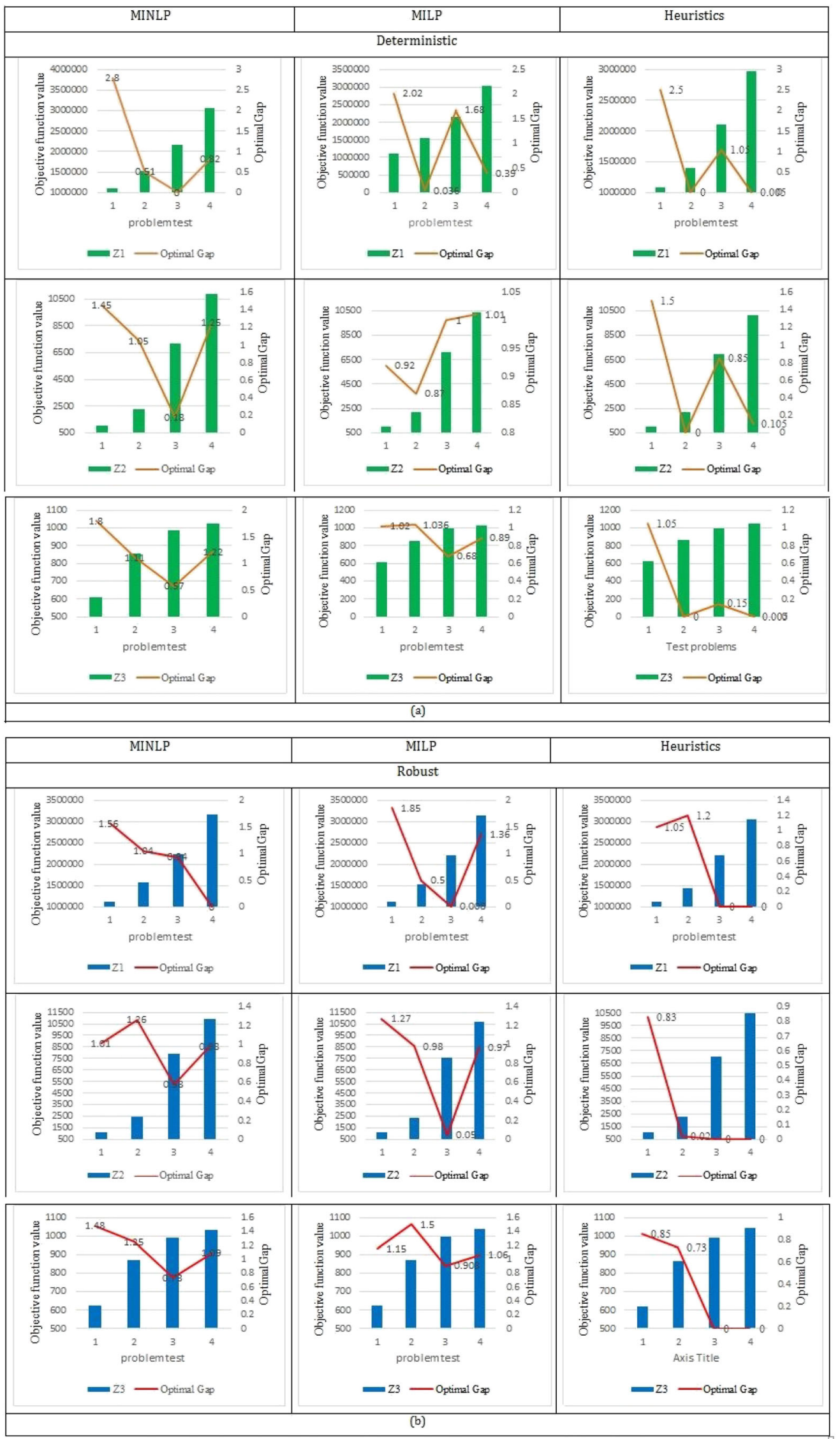

Fig. 2 Comparison of different solution methods in uncertainty and deterministic conditions 
$50 \%$. On the other hand, the environmental impact has increased by $62 \%$, which can be due to increased transportation, heavy traffic, and product production with shelf time is lower. But as you know, increased demand has a direct relationship with customer satisfaction, which increases by almost $80 \%$.
Fig. 3 Comparison of the results for case study problem in deterministic and robust situation

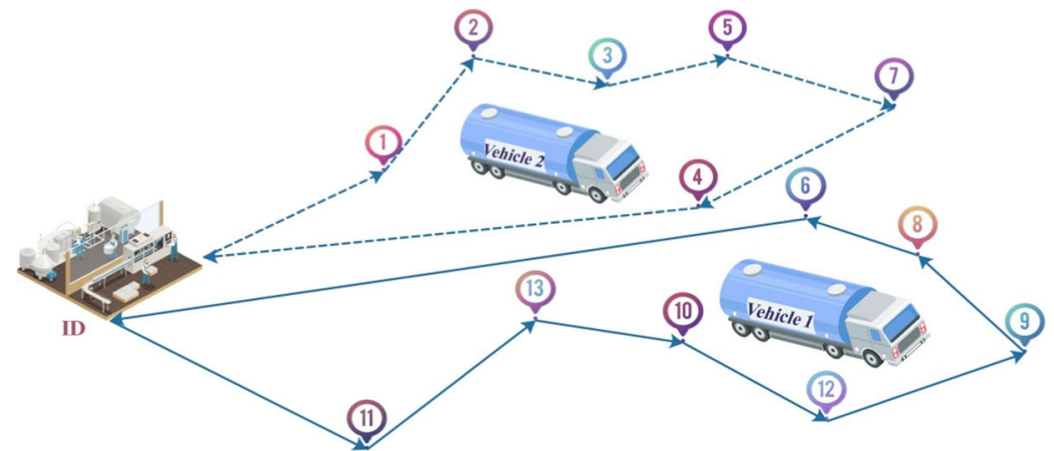

(a)

1 vehicle's trip =ID-11-13-10-12-9-8-6-ID

2 vehicle's trip =ID-1-2-3-5-7-4-ID

The objective functions: cost=1488040; Environmental impacts=1193; customer satisfaction=823

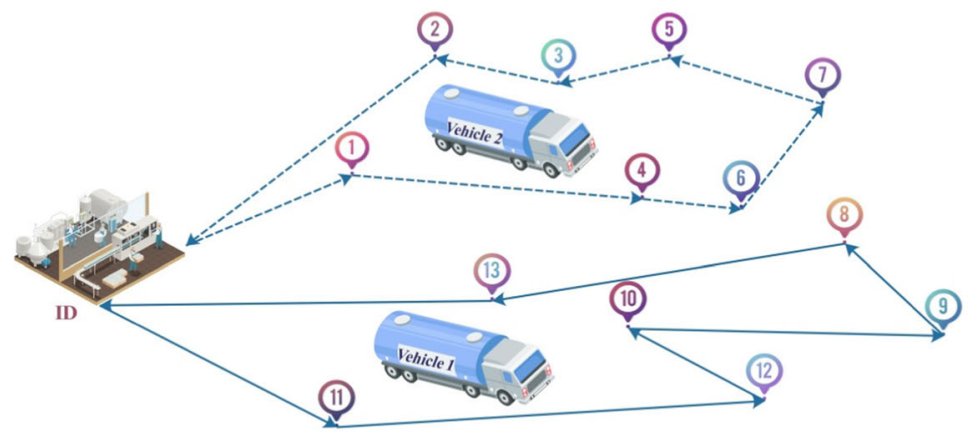

(b)

1 vehicle's trip =ID-11-12-10-9-8-13-ID

2 vehicle's trip =ID-1-4-6-7-5-3-2-ID

The objective functions: cost=1300141; Environmental impacts=1103; customer satisfaction=805

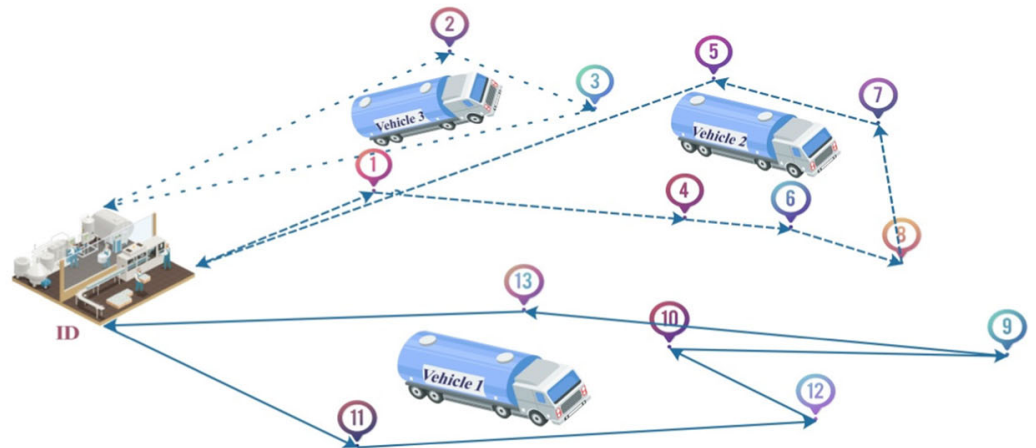

(c)

1 vehicle's trip =ID-11-12-10-9-8-13-ID

2 vehicle's trip =ID-1-4-6-7-5-ID

3 vehicle's trip =ID-2-3-ID

The objective functions: cost=1572005; Environmental impacts=1243; customer satisfaction=875 
Fig. 4 Comparison of the results for case study problem with considering traffic and non-traffic conditions in deterministic and robust situation

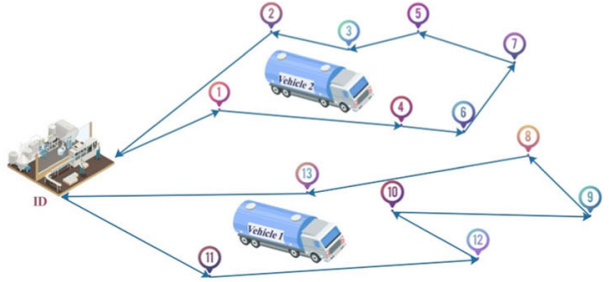

(a-1)

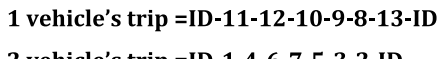

2 vehicle's trip =ID-1-4-6-7-5-3-2-ID

The objective functions: cost=1300141; Environmental impacts=1103; customer satisfaction $=\mathbf{8 0 5}$

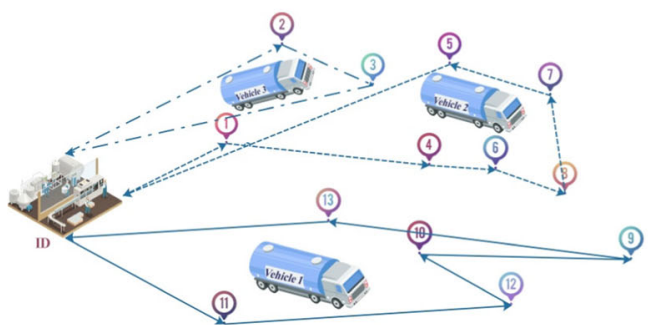

(b-1)

1 vehicle's trip =ID-11-12-10-9-8-13-ID

2 vehicle's trip =ID-1-4-6-7-5-ID

3 vehicle's trip =ID-2-3-ID

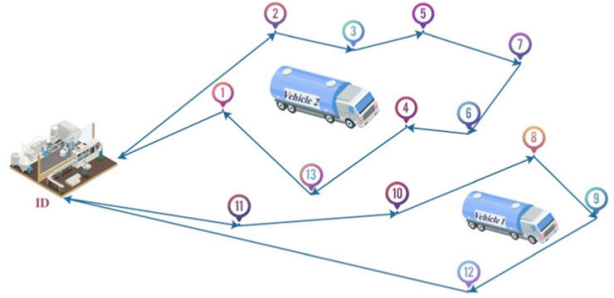

(a-2)

condition(Deterministic)

1 vehicle's trip =ID-11-10-8-9-12-ID

2 vehicle's trip =ID-2-3-5-7-6-4-13-ID

The objective functions: cost=1219341; Environmental impacts=1095; customer satisfaction $=725$

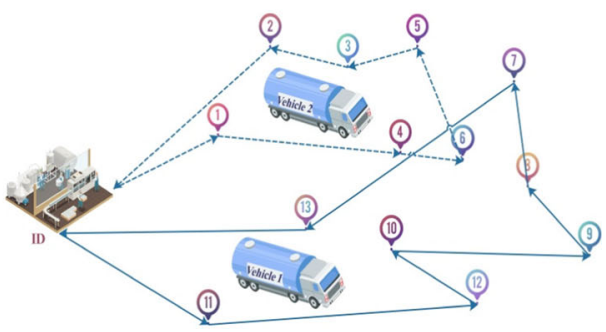

(b-2)

1 vehicle's trip =ID-11-12-10-9-8-7-13-ID

2 vehicle's trip =ID-1-4-6-5-3-2-ID

The objective functions: $\cos t=1115405$; Environmental impacts=995; customer satisfaction $=614$
The objective functions: cost=1572005; Environmental impacts=1243; customer satisfaction $=875$

\section{Shelf time}

In the account of the various lifespans of products and their significance in environmental pollution, this factor takes one to reduce the costs of logistics. Hence, production managers in the dairy supply chain must adopt decisions in the production system with the greatest durability and minimal environmental pollution, which will always arrive at economic growth in this industry. Figure 7 showed the results of the sensitivity analysis of the lifetime of the product 2 to 5 weeks. The total costs and environmental impacts were decreased about 38\% and $18 \%$, respectively and customer satisfaction was increased about $22 \%$.

\section{Traffic conditions}

In this section, the sensitivity analysis of the objective functions is comparable to the diverse traffic conditions of the model to specify the effect of the variation in the value of the objective functions. The impression of traffic conditions,
Fig. 5 Comparison of the results for different optimal status

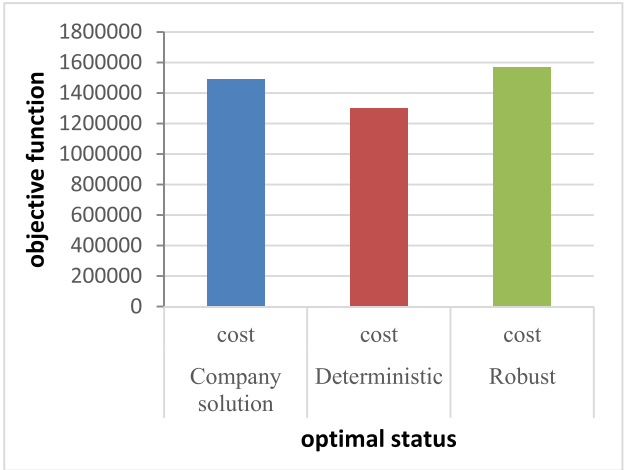

(a)

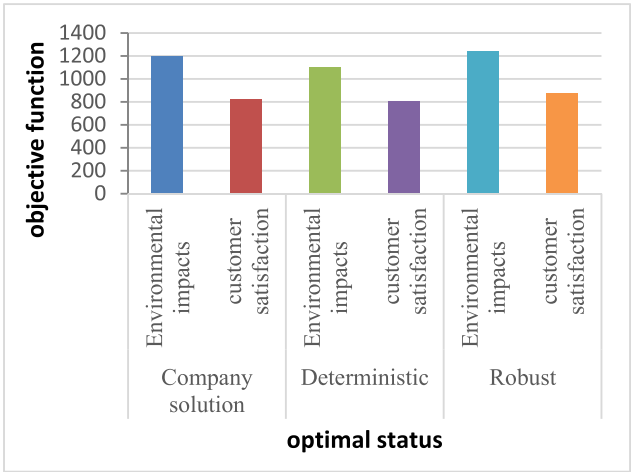

(b) 
Fig. 6 Demand sensitivity analysis on objective functions

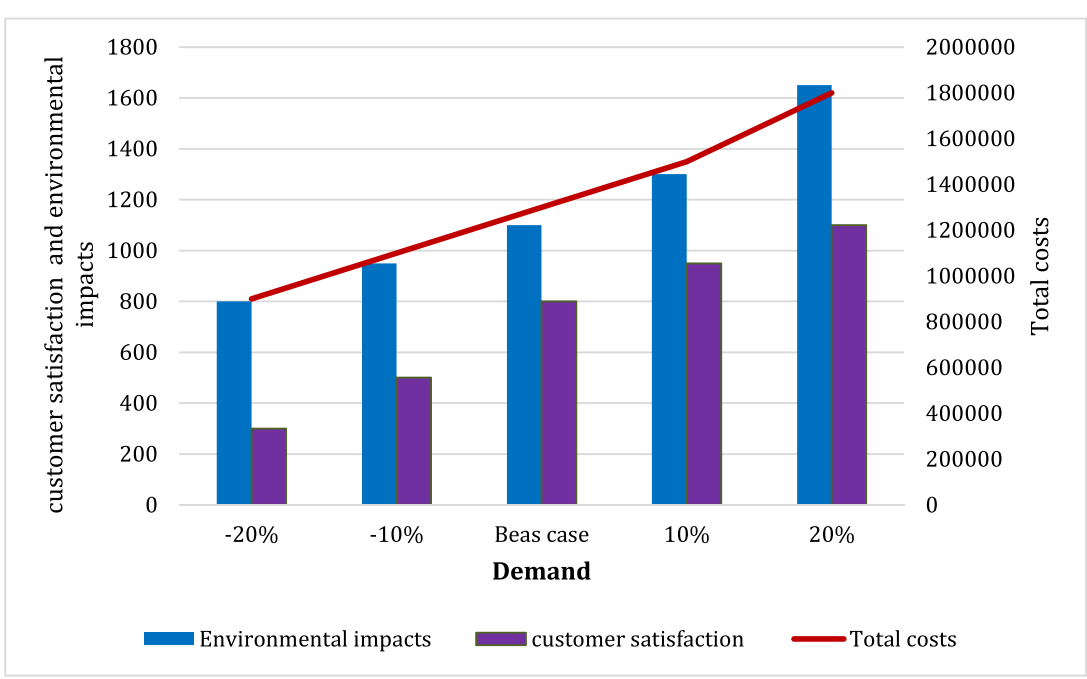

as referred to, will accidentally affect the speed of the vehicle and the amount of travel time, which will have a complete impact on costs. As far as you can see in Fig. 8, the application of traffic conditions and the non-application of traffic conditions increase or decrease the objective functions. This indicates the momentousness of traffic conditions and planning at the procurement and transportation levels, and additionally demonstrates the capability of the proposed algorithm to tackle dynamic changes with the advancement of various configurations for use at diverse decision points.

As you can see, when we move from non-traffic to heavy traffic, there will be a $43 \%$ increase in total cost and $38 \%$ increase in environmental impact. In addition, customer satisfaction will be reduced by $70 \%$.

\section{Managerial implications}

According to the results of the analysis in Section 5, transportation system managers can make important decisions about traffic conditions and customer service, and stochastic travel times in different scenarios. Dynamic stochastic models can have important implications for managers' decisions to make integrated decisions about financial flows in uncertain markets and optimal flows in optimal routes to serve customers.

Managers can benefit from the model outputs by using these models and creating optimal facilities for transportation in assessing the traffic conditions of the routes, in connection with the optimal route allocation to customers, effective decisions in the transportation industry. Since dynamism in the transportation system is inevitable, it is necessary to be prepared to face different scenarios in the transportation system. In some cases, the interaction between scenarios is important. Consider, traffic increases, costs increase, and customer dissatisfaction happens at the same time. Then, the manager must know what degree (feature) of the route, such as the type of diversion routes and how long to reach the destination and with what traffic conditions to maximize customer satisfaction and increase revenue, pay attention that at the same time overall costs are rising. Also, due to the speed of fluctuations, the level of dynamism also changes, leading to the nature of quick decisions to maintain a competitive market.
Fig. 7 Sensitivity analysis of shelf time of products in different objective functions

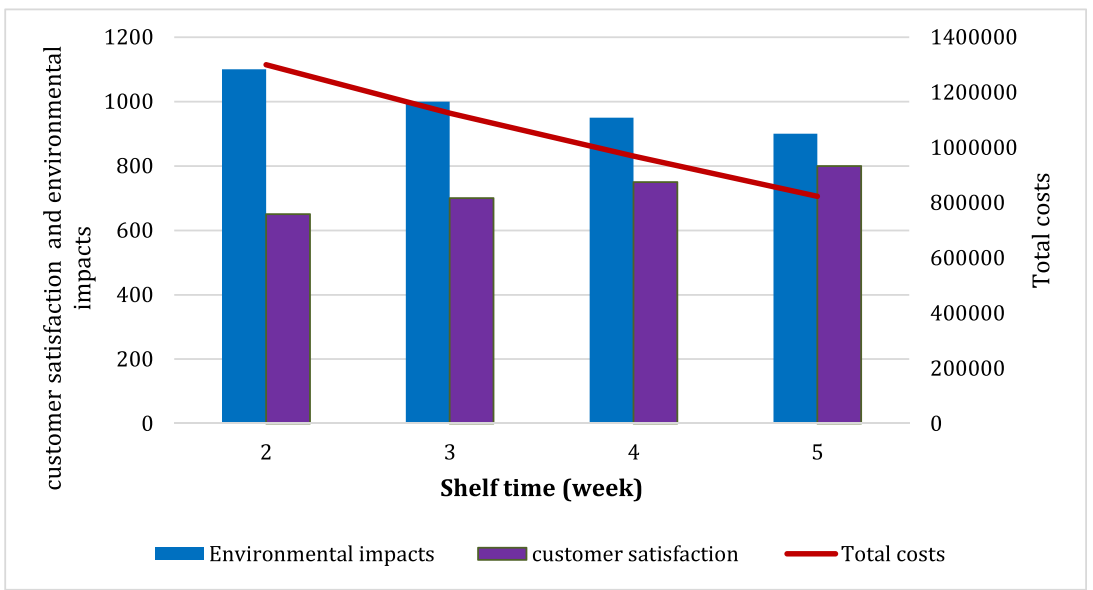


Fig. 8 Sensitivity analysis of traffic conditions in different objective functions

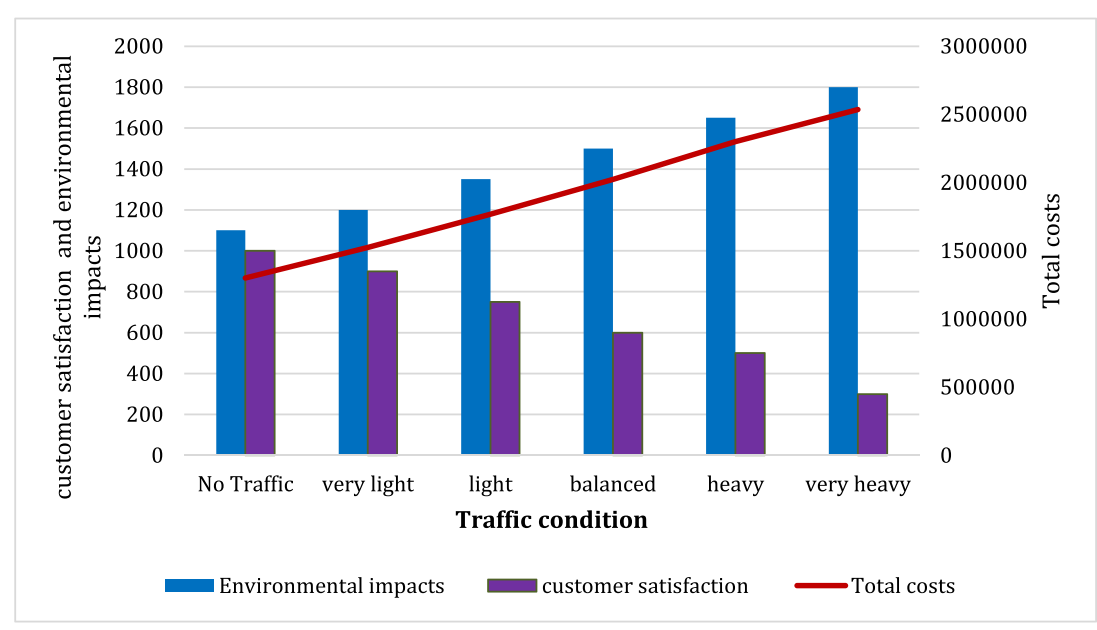

Improving the optimal route allocation for the transport of perishable products significantly changes the allocation of traffic flow, but does not necessarily lead to improved efficiency (time savings) in the transportation system. This is because the greater the value of perishable products with a longer lifespan in different scenarios, the greater the cost reduction of existing heterogeneous shipments. This leads to a turning point and homogeneous selective behavior for managers. As a result, managers can improve the efficiency (less travel time) of the transportation system by making long-term decisions to extend the life of perishable products with different milestones (lower cost of optimal route allocation).

\section{Conclusion}

In this study, in order that optimizing logistics systems and system efficiency to minimize the total cost for a dynamic transport network and to minimize carbon emissions and maximize the level of customer service, an improved optimization model with the Logit function been fulfilled to achieve the probability and allocation of routes owing to the various green traffic conditions for perishable products under uncertainty. Hence, in this paper, dynamic vehicle routing to accompany the customer service time window is restricted to the process of vehicle implementation in a logistics transportation system in a case study in Kaleh Dairy Company in Iran. In this case, owing to various traffic conditions, vehicle speed and travel time are taken into consideration without prior planning. In order that solves the proposed model, this paper has been formulated a heuristic hybrid optimization method with the $\varepsilon$-constraint method and to deal with the uncertainty in demand for robust optimization in 4 different sizes. Afterward, the optimal routing established upon the proposed method for the case study is demonstrated. Sensitivity analysis for traffic conditions and product longevity was also carried out for optimal policy decisions. The results showed that the proposed model improves the company's costs by $12.6 \%$ in the deterministic model, also improves the environmental effects by $7.5 \%$ and customer satisfaction by $2.18 \%$. However, we are in robust mode due to the existence of a penalty cost with a relatively small increase in costs. Although the results of the calculations represented efficacious results, in order to improve these results, further development of the scenarios should be evaluated, which can be fulfilled for future research.

Suggestions for future research, containing integration of uncertainty in problem parameters and fuzzy scenario-based optimization approaches, as well as the use of meta-heuristic algorithms and machine learning, could be taken into account as future challenges.

Author contribution Reza Zakaryaei Talouki: conceptualization, methodology, software, formal analysis, writing-original draft, investigation, data curation. Nikbakhsh Javadian: supervisor, review \& editing, resources. Mohammad Mehdi Movahedi: validation, writing.

Data availability All data is available in the paper.

\section{Declarations}

Consent for publication The authors agree to publish.

Conflict of interest The authors declare no competing interests.

\section{References}

Alinaghian M, Tirkolaee EB, Dezaki ZK, Hejazi SR, Ding W (2020) An augmented Tabu search algorithm for the green inventory-routing problem with time windows. Swarm Evol Comput 60:100802

Alkaabneh F, Diabat A, Gao HO (2020) Benders decomposition for the inventory vehicle routing problem with perishable products and environmental costs. Comput Oper Res 113:104751

Amorim P, Almada-Lobo B (2014) The impact of food perishability issues in the vehicle routing problem. Comput Ind Eng 67:223-233 
Anderluh A, Nolz PC, Hemmelmayr VC, Crainic TG (2019) Multiobjective optimization of a two-echelon vehicle routing problem with vehicle synchronization and 'grey zone' customers arising in urban logistics. Eur J Oper Res

Babaee Tirkolaee E, Abbasian P, Soltani M, Ghaffarian SA (2019) Developing an applied algorithm for multi-trip vehicle routing problem with time windows in urban waste collection: a case study. Waste Manag Res 37(1 suppl):4-13

Bairamzadeh S, Saidi-Mehrabad M, Pishvaee MS (2018) Modelling different types of uncertainty in biofuel supply network design and planning: a robust optimization approach. Renew Energy 116: 500-517

Bouziyane B, Dkhissi B, Cherkaoui M (2018) Solving a dynamic vehicle routing problem with soft time windows based on static problem resolution by a hybrid approach. Int J Supply Oper Manag 5(2): 134-151

Bruglieri M, Mancini S, Pezzella F, Pisacane O (2019) A path-based solution approach for the Green Vehicle Routing Problem. Comput Oper Res 103:109-122

Buhler, G. \& Jochem, P. (2008). CO2 reductions in freight transports: how to stimulate environmental friendly behaviour? Centre for European Economic Research. Discussion paper no.

Ćirović G, Pamučar D, Božanić D (2014) Green logistic vehicle routing problem: routing light delivery vehicles in urban areas using a neuro-fuzzy model. Expert Syst Appl 41(9):4245-4258

Dantzig, G. B., \& Ramser, J. H. (1959). The truck dispatching problem. Manag Sci, 6(1), 80-91. Delivery vehicles in urban areas using a neuro-fuzzy model. Expert Syst Appl. 41 (9), 4245e4258.

Dunn JB, Gaines L, Sullivan J, Wang MQ (2012) Impact of recycling on cradle-to-gate energy consumption and greenhouse gas emissions of automotive lithium-ion batteries. Environ Sci Technol 46(22): 12704-12710

Erdoğan S, Miller-Hooks E (2012) A green vehicle routing problem. Transp Res Part E: Logist Transp Rev 48(1):100-114

Franceschetti A, Demir E, Honhon D, Van Woensel T, Laporte G, Stobbe M (2017) A metaheuristic for the time-dependent pollutionrouting problem. Eur J Oper Res 259(3):972-991

Gaines, L., Rask, E., \& Keller, G. (2013). Which is greener: idle, or stop and restart? Comparing fuel use and emissions for short passengercar stops (No. 13-4606).

Gholizadeh H, Fazlollahtabar H (2020) Robust optimization and modified genetic algorithm for a closed loop green supply chain under uncertainty: case study in melting industry. Comput Ind Eng: 106653

Gholizadeh H, Fazlollahtabar H, Khalilzadeh M (2020) A robust fuzzy stochastic programming for sustainable procurement and logistics under hybrid uncertainty using big data. J Clean Prod: 120640

Gholizadeh H, Tajdin A, Javadian N (2020) A closed-loop supply chain robust optimization for disposable appliances. Neural Comput Applic 32(8):3967-3985

Giallanza A, Puma GL (2020) Fuzzy green vehicle routing problem for designing a three echelons supply chain. J Clean Prod 259:120774

Govindan K, Jafarian A, Khodaverdi R, Devika K (2014a) Two-echelon multiple-vehicle location-routing problem with time windows for optimization of sustainable supply chain network of perishable food. Int J Prod Econ 152:9-28

Govindan K, Jafarian A, Khodaverdi R, Devika K (2014b) Two-echelon multiple-vehicle location-routing problem with time windows for optimization of sustainable supply chain network of perishable food. Int J Prod Econ 152:9-28

Herbon A, Khmelnitsky E (2017) Optimal dynamic pricing and ordering of a perishable product under additive effects of price and time on demand. Eur J Oper Res 260(2):546-556

Huang Y-H, Blazquez CA, Huang S-H, Paredes-Belmar G, LatorreNuñez G (2019) Solving the feeder vehicle routing problem using ant colony optimization. Comput Ind Eng 127:520-535
Jabali O, Woensel T, de Kok A (2012) Analysis of travel times and CO2 emissions in time-dependent vehicle routing. Prod Oper Manag 21(6):1060-1074

Jabbarpour MR, Noor RM, Khokhar RH (2015) Green vehicle traffic routing system using ant-based algorithm. J Netw Comput Appl 58:294-308

Jouzdani J, Sadjadi SJ, Fathian M (2013) Dynamic dairy facility location and supply chain planning under traffic congestion and demand uncertainty: a case study of Tehran. Appl Mathe Model 37(18-19): $8467-8483$

Keyvanshokooh E, Ryan SM, Kabir E (2016) Hybrid robust and stochastic optimization for closed-loop supply chain network design using accelerated Benders decomposition. Eur J Oper Res 249(1):76-92

Kim G, Ong YS, Cheong T, Tan PS (2016) Solving the dynamic vehicle routing problem under traffic congestion. IEEE Transact Intell Transp Syst 17(8):2367-2380

Koc C, Karaoglan I (2016) The green vehicle routing problem: a heuristic based exact solution approach. Appl Soft Comput 39:154-164

Koyuncu I, Yavuz M (2019) Duplicating nodes or arcs in green vehicle routing: a computational comparison of two formulations. Transp Res Part E: Logist Transp Rev 122:605-623

Kwon YJ, Choi YJ, Lee DH (2013) Heterogeneous fixed fleet vehicle routing considering carbon emission. Transp Res Part D: Transp Environ 23:81-89

Kucukoğlu İ, Ene S, Aksoy A, Ozturk N (2015) A memory structure adapted simulated annealing algorithm for a green vehicle routing problem. Environ Sci Pollut Res 22(5):3279-3297

Li Y, Lim MK, Tseng ML (2019) A green vehicle routing model based on modified particle swarm optimization for cold chain logistics. Ind Manag Data Syst

Lin C, Choy KL, Ho GT, Chung SH, Lam HY (2014) Survey of green vehicle routing problem: past and future trends. Expert Syst Appl 41(4):1118-1138

Ma ZJ, Wu Y, Dai Y (2017) A combined order selection and timedependent vehicle routing problem with time widows for perishable product delivery. Comput Ind Eng 114:101-113

Macrina G, Laporte G, Guerriero F, Di Puglia Pugliese L (2019a) An energy-efficient green-vehicle routing problem with mixed vehicle fleet, partial battery recharging and time windows. Eur J Oper Res 276(3):971-982

Macrina G, Laporte G, Guerriero F, Di Puglia Pugliese L (2019b) An energy-efficient green-vehicle routing problem with mixed vehicle fleet, partial battery recharging and time windows. Eur J Oper Res 276(3):971-982

Marcilio GP, de Assis Rangel JJ, de Souza CLM, Shimoda E, da Silva FF, Peixoto TA (2018) Analysis of greenhouse gas emissions in the road freight transportation using simulation. J Clean Prod 170:298309

Niu Y, Yang Z, Chen P, Xiao J (2018) Optimizing the green open vehicle routing problem with time windows by minimizing comprehensive routing cost. J Clean Prod 171:962-971

Pérez-Rodríguez R, Hernández-Aguirre A (2019) A hybrid estimation of distribution algorithm for the vehicle routing problem with time windows. Comput Ind Eng 130:75-96

Poonthalir G, Nadarajan R (2018) A fuel efficient green vehicle routing problem with varying speed constraint (F-GVRP). Expert Syst Appl 100:131-144

Pradenas L, Oportus B, Parada V (2013) Mitigation of greenhouse gas emissions in vehicle routing problems with backhauling. Expert Syst Appl 40(8):2985-2991

Qian J, Eglese R (2016) Fuel emissions optimization in vehicle routing problems with time-varying speeds. Eur J Oper Res 248(3):840-848

Rahbari A, Nasiri MM, Werner F, Musavi M, Jolai F (2019) The vehicle routing and scheduling problem with cross-docking for perishable products under uncertainty: two robust bi-objective models. Appl Math Model 70:605-625 
Rahimi, M., Baboli, A., \& Rekik, Y. (2017). Inventory routing problem for perishable products by considering customer satisfaction and green criteria. In Dynamics in Logistics (pp. 445-455). Springer, Cham.

Rahimi M, Baboli A, Rekik Y (2017) Multi-objective inventory routing problem: a stochastic model to consider profit, service level and green criteria. Transport Res E-Log 101:59-83

Razavi N, Gholizadeh H, Nayeria S, Ashrafi TA (2020) A robust optimization model of the field hospitals in the sustainable blood supply chain in crisis logistics. J Oper Res Soc:1-26

Rezaei-Malek M, Tavakkoli-Moghaddam R, Zahiri B, Bozorgi-Amiri A (2016) An interactive approach for designing a robust disaster relief logistics network with perishable commodities. Comput Ind Eng 94: 201-215

Rezgui D, Chaouachi Siala J, Aggoune-Mtalaa W, Bouziri H (2019) Application of a variable neighborhood search algorithm to a fleet size and mix vehicle routing problem with electric modular vehicles. Comput Ind Eng 130:537-550

Sim J (2017) The influence of new carbon emission abatement goals on the truck-freight transportation sector in South Korea. J Clean Prod 164:153-162

Solano-Charris E, Prins C, Santos AC (2015) Local search based metaheuristics for the robust vehicle routing problem with discrete scenarios. Appl Soft Comput 32:518-531

Soleimani H, Chaharlang Y, Ghaderi H (2018) Collection and distribution of returned-remanufactured products in a vehicle routing problem with pickup and delivery considering sustainable and green criteria. J Clean Prod 172:960-970

Song BD, Ko YD (2016) A vehicle routing problem of both refrigeratedand general-type vehicles for perishable food products delivery. J Food Eng 169:61-71

Soysal M, Çimen M (2017) A simulation based restricted dynamic programming approach for the green time dependent vehicle routing problem. Comput Oper Res 88:297-305

Soysal M, Cimen M, Demir E (2018) On the mathematical modeling of green one-to-one pickup and delivery problem with road segmentation. J Clean Prod 174:1664-1678

Soysal M, Bloemhof-Ruwaard JM, Haijema R, van der Vorst JG (2018) Modeling a green inventory routing problem for perishable products with horizontal collaboration. Comput Oper Res 89:168-182

Sureeyatanapas P, Poophiukhok P, Pathumnakul S (2018) Green initiatives for logistics service providers: an investigation of antecedent factors and the contributions to corporate goals. J Clean Prod 191:114

Suzuki Y, Kabir QS (2015) Green vehicle routing for small motor carriers. Transp J 54(2):186-212

Taș D, Gendreau M, Dellaert N, van Woensel T, de Kok AG (2014) Vehicle routing with soft time windows and stochastic travel times: a column generation and branch-and-price solution approach. Eur J Oper Res 236(3):789-799
Tirkolaee EB, Abbasian P, Weber GW (2020) Sustainable fuzzy multitrip location-routing problem for medical waste management during the COVID-19 outbreak. Sci Total Environ:143607

Tirkolaee EB, Goli A, Faridnia A, Soltani M, Weber GW (2020) Multiobjective optimization for the reliable pollution-routing problem with cross-dock selection using Pareto-based algorithms. J Clean Prod 276:122927

Tirkolaee EB, Hadian S, Weber GW, Mahdavi I (2020) A robust green traffic-based routing problem for perishable products distribution. Comput Intell 36(1):80-101

Tiwari A, Chang P-C (2015) A block recombination approach to solve green vehicle routing problem. Int J Prod Econ 164:379-387

Utama DM, Dewi SK, Wahid A, Santoso I (2020) The vehicle routing problem for perishable goods: a systematic review. Cogent Eng 7(1): 1816148

Vidal T, Crainic TG, Gendreau M, Prins C (2013) Heuristics for multiattribute vehicle routing problems: a survey and synthesis. Eur J Oper Res 231(1):1-21

Vornhusen B, Kopfer H (2015) Emission vehicle routing problem with split delivery and a heterogeneous vehicle fleet. Comput Logist:7690

Xiao Y, Konak A (2015) A simulating annealing algorithm to solve the green vehicle routing \& scheduling problem with hierarchical objectives and weighted tardiness. Appl Soft Comput 34:372-388

Xiao Y, Konak A (2016) The heterogeneous green vehicle routing and scheduling problem with time-varying traffic congestion. Transport Res E-Log 88:146-166

Xiao Y, Konak A (2017) A genetic algorithm with exact dynamic programming for the green vehicle routing \& scheduling problem. J Clean Prod 167(Suppl. C):1450e1463

Xiao X, Yan Y, Chen B (2019) Stochastic dynamic analysis for vehicletrack-bridge system based on probability density evolution method. Eng Struct 188:745-761

Xu Z, Elomri A, Pokharel S, Mutlu F (2019) A model for capacitated green vehicle routing problem with the time-varying vehicle speed and soft time windows. Comput Ind Eng 137:106011

Yavari M, Geraeli M (2019) Heuristic method for robust optimization model for green closed-loop supply chain network design of perishable goods. J Clean Prod 226:282-305

Yin P-Y, Chuang Y-L (2016) Adaptive memory artificial bee colony algorithm for green vehicle routing with cross-docking. Appl Math Modell 40(21):9302-9315

Zhang J, Zhao Y, Xue W, Li J (2015) Vehicle routing problem with fuel consumption and carbon emission. Int J Prod Econ 170:234-242

Zulvia FE, Kuo RJ, Nugroho DY (2020) A many-objective gradient evolution algorithm for solving a green vehicle routing problem with time windows and time dependency for perishable products. J Clean Prod 242:118428

Publisher's note Springer Nature remains neutral with regard to jurisdictional claims in published maps and institutional affiliations. 